\title{
Article \\ Surgical Resection vs. Percutaneous Ablation for Single Hepatocellular Carcinoma: Exploring the Impact of Li-RADS Classification on Oncological Outcomes
}

\author{
Leonardo Centonze ${ }^{1, *(\mathbb{D}}$, Stefano Di Sandro ${ }^{1,2}$, Andrea Lauterio ${ }^{1} \mathbb{D}^{\mathbb{D}}$, Riccardo De Carlis ${ }^{1} \mathbb{D}$, Samuele Frassoni ${ }^{3}$, \\ Antonio Rampoldi ${ }^{4}$, Bruno Tuscano ${ }^{4}$, Vincenzo Bagnardi ${ }^{3}$, Angelo Vanzulli ${ }^{4}\left(\mathbb{D}\right.$ and Luciano De Carlis ${ }^{1,5}$ \\ 1 Department of General Surgery and Transplantation, Niguarda Ca' Granda Hospital, 20162 Milan, Italy; \\ stefano.disandro@unimore.it (S.D.S.); andrea.lauterio@ospedaleniguarda.it (A.L.); \\ riccardo.decarlis@ospedaleniguarda.it (R.D.C.); luciano.decarlis@ospedaleniguarda.it (L.D.C.) \\ 2 Hepato-Pancreato-Biliary Surgery and Liver Transplantation Unit, University of Modena and Reggio Emilia, \\ 41124 Modena, Italy \\ 3 Department of Statistics and Quantitative Methods, University of Milan-Bicocca, 20126 Milan, Italy; \\ samuele.frassoni@unimib.it (S.F.); vincenzo.bagnardi@gmail.com (V.B.) \\ 4 Department of Diagnostic and Interventional Radiology, Niguarda Ca' Granda Hospital, 20162 Milan, Italy; \\ antoniogaetano.rampoldi@ospedaleniguarda.it (A.R.); bruno.tuscano@ospedaleniguarda.it (B.T.); \\ angelo.vanzulli@ospedaleniguarda.it (A.V.) \\ check for \\ updates \\ 5 School of Medicine and Surgery, University of Milan-Bicocca, 20126 Milan, Italy \\ * Correspondence: leonardo.centonze@ospedaleniguarda.it; Tel.: +39-02-6444-4895
}

Citation: Centonze, L.; Di Sandro, S.; Lauterio, A.; De Carlis, R.; Frassoni, S.; Rampoldi, A.; Tuscano, B.; Bagnardi, V.; Vanzulli, A.; De Carlis, L. Surgical Resection vs. Percutaneous Ablation for Single Hepatocellular Carcinoma: Exploring the Impact of Li-RADS Classification on Oncological Outcomes. Cancers 2021, 13, 1671. https://doi.org/ 10.3390/cancers13071671

Academic Editors: Christoph Reissfelder and Yasunori Minami

Received: 25 January 2021

Accepted: 31 March 2021

Published: 1 April 2021

Publisher's Note: MDPI stays neutral with regard to jurisdictional claims in published maps and institutional affiliations.

Copyright: (c) 2021 by the authors. Licensee MDPI, Basel, Switzerland. This article is an open access article distributed under the terms and conditions of the Creative Commons Attribution (CC BY) license (https:// creativecommons.org/licenses/by/ $4.0 /)$.
Simple Summary: Li-RADS classification has recently emerged as an accurate tool for hepatocellular carcinoma diagnosis in the setting of liver cirrhosis, but its prognostic value has never been investigated so far. Single HCC benefits from both surgical resection and percutaneous ablation, although several studies support the superiority of surgery in terms of oncological results. We retrospectively and blindly classified 140 treatment-naïve single HCC according to Li-RADS protocol, comparing the oncological outcomes of surgical resection and percutaneous ablation for each Li-RADS subclass. Our analysis highlighted better overall survival, recurrence free survival and lower incidence of local recurrence after surgical resection in Li-RADS-5 nodules, while Li-RADS-3/4 subclasses showed similar outcomes after the two treatments. These results confirm the superiority of surgical approach for single HCC and suggest a potential prognostic role of Li-RADS classification, supporting liver resection especially for Li-RADS-5 subclass.

\begin{abstract}
Background: Single hepatocellular carcinoma (HCC) benefits from surgical resection (SR) or US-guided percutaneous ablation (PA), although the best approach is still debated. We evaluated the impact of Li-RADS classification on the oncological outcomes of SR vs. PA as single HCC first-line treatment. Methods: We retrospectively and blindly classified treatment-naïve single HCC that underwent SR or PA between 2010 and 2016 according to Li-RADS protocol. Overall survival (OS), recurrence free survival (RFS) and local recurrence after SR and PA were compared for each Li-RADS subclass before and after propensity-score matching (PS-M). Results: Considering the general population, SR showed better 5 -year OS (68.3\% vs. 52.2\%; $p=0.049)$ and RFS $(42.5 \%$ vs. $29.8 \%$; $p=0.002)$, with lower incidence of local recurrence ( $8.2 \%$ vs. $44.4 \% ; p<0.001)$, despite a significantly higher frequency of clinically-relevant complications $(12.8 \%$ vs. $1.9 \% ; p=0.002)$ and a higher Comprehensive Complication Index (12.1 vs. 2.2; $p<0.001)$. Focusing on different Li-RADS subclasses, we highlighted better 5 -year OS (67.1\% vs. $46.2 \% ; p=0.035)$, RFS (45.0\% vs. $27.0 \%$ RFS; $p<0.001)$ and lower incidence of local recurrence $(9.7 \%$ vs. $48.6 \% ; p<0.001)$ after SR for Li-RADS-5 HCCs, while these outcomes did not differ for Li-RADS-3/4 subclasses; such results were confirmed after PS-M. Conclusions: Our analysis suggests a potential prognostic role of Li-RADS classification, supporting SR over PA especially for Li-RADS-5 single HCC.
\end{abstract}


Keywords: hepatocellular carcinoma; Li-RADS classification; surgical resection; percutaneous ablation; recurrence; survival

\section{Introduction}

Hepatocellular carcinoma (HCC) represents the third cause of cancer-related death worldwide accounting for $85-90 \%$ of primary liver tumors, and is associated to liver cirrhosis in more than $80 \%$ of patients [1-3].

Up to the most recent guidelines from the European Association for the Study of the Liver (EASL) and the American Association for the Study of Liver Disease (AASLD), HCC diagnosis in the setting of liver cirrhosis relies on contrast-enhanced imaging [4,5].

American Association of Radiologist developed a standardized diagnostic algorithm for imaging reporting in the setting of liver cirrhosis: Liver Reporting \& Data System (Li-RADS) [5]. The Li-RADS protocol provides HCC-specific diagnostic accuracy for each Li-RADS subclass, ranging from 37\% in Li-RADS-3 up to 95\% in Li-RADS-5 nodules [6,7], and has been recently included in AASLD guidelines for HCC management [5].

HCC treatment algorithms are deeply influenced by the correlation of HCC and underlying liver cirrhosis, as treatment options may be limited by the reduced physiological reserve of a diseased liver, as well as tumor burden.

Single HCC could benefit from both surgical resection (SR) or US-guided percutaneous ablation (PA) with similar oncological outcomes [8-10], although several studies advocate resection as the best treatment option [11-13]; notably, pathological analysis from surgical specimens could reveal the presence of unfavorable histological characteristics such as microvascular invasion or satellitosis, that may change future clinical decision making (i.e., favoring liver transplantation) [14-16]. Given this scenario, the aim of our study was to retrospectively evaluate the oncological impact of Li-RADS classification on single HCC treated by SR vs. PA.

\section{Materials and Methods}

Study protocol followed the 1975 Declaration of Helsinki ethical guidelines, as revised in Brazil in 2013. Local ethical committees' review of the protocol deemed that formal approval was not required owing to the retrospective, observational and anonymous nature of this study. Results are reported according to Strengthening the Reporting of Observational Studies in Epidemiology (STROBE) [17].

\subsection{Study Design}

The study enrolled all adult patients (age $\geq 18$ years) with a history of cirrhosis and a treatment-naïve single HCC without macrovascular invasion who underwent SR or PA as first-line treatment between 2010 and 2016, with an available pre-treatment contrastenhanced imaging and $>1$-year follow-up.

All data were retrieved from a single university-affiliated, hepato-pancreato-biliary teaching center prospective database and anonymized prior to analysis.

A senior (A.V.) and a fellow (B.T.) radiologist retrospectively and blindly analyzed pretreatment contrast-enhanced imaging (either computed tomography or magnetic resonance imaging) in order to classify all nodules according to 2018 Li-RADS protocol, applying ancillary features when feasible [6]. HCCs whose edge was located $5 \mathrm{~mm}$ or less from the surface of the liver were defined as superficial.

Oncological outcomes of SR and PA were compared before and after Li-RADS stratification of the general population for Li-RADS- 5 and Li-RADS-3/4 subclasses; such results were furtherly validated after propensity-score matching (PS-M). 


\subsection{Perioperative Management}

HCC diagnosis followed EASL and AASLD guidelines [4,5]. All treatment options were discussed during multidisciplinary institutional boards composed by surgeons, hepatologists, diagnostic and interventional radiologists.

Impaired hepatic functional reserve (Child Pugh score C, ascites, platelet count $<50.000 / \mathrm{uL}$ ) and high operative risk (ASA score $>3$ ) were considered as contraindications to surgery; on the other hand, a peripheral exophytic lesion and a nodule diameter $>5 \mathrm{~cm}$ or not detectable by ultrasound usually contraindicated ablation.

Postoperative or postprocedural complications were recorded according to ClavienDindo classification, and summarized in the Comprehensive Complication Index (CCI) $[18,19]$, while post-operative biliary fistula and post-hepatectomy liver failure were defined following the International Study Group for Liver Surgery (ISGLS) classifications [20,21].

Liver transplantation was not considered as first line approach for early HCC, but represented the leading option in patients who experienced a transplantable recurrence [22]. All patients underwent life-long surveillance for HCC recurrence, and local recurrence was diagnosed and evaluated following the EASL guidelines [4].

\subsection{Study Endopints}

The endpoints of our study were recurrence-free survival (RFS), defined as the time from surgery or ablation until the date of any type of HCC recurrence, either local or distant, and overall survival (OS), defined as the time from surgery until the date of death, all causes considered.

\subsection{Statistical Analysis}

Continuous data are reported as median and interquartile ranges (IQR). Categorical data are reported as counts and percentages.

Comparisons between resected and ablated HCC patients were performed using the Wilcoxon test for continuous variables and Chi-square test for categorical variables.

Given the differences in the baseline characteristics between resected and ablated populations, and to control nonrandom assignment of the treatment, a second analysis was performed on a subset of all patients considered in the study, according to a PS-M.

The PS-M was estimated with the use of a multivariable logistic regression model, with treatment as the dependent variable and the following baseline characteristics as covariates: diameter of the nodule, alpha-fetoprotein (a-FP), satellitosis and platelet count. PS-M was performed with the use of a 1:1 matching without replacement (greedy-matching algorithm), with a caliper width equal to 0.30 of the propensity score. Comparisons between the two matched groups were performed using the Wilcoxon test for continuous variables and Fisher's exact test for categorical variables.

The RFS and OS functions were estimated using the Kaplan-Meier method, and the log-rank test was performed to evaluate differences between groups.

The cumulative incidence functions (CIF) of local relapse were estimated according to method described by Kalbfleisch and Prentice, taking into account the competing causes of recurrence. Gray's test was used to assess differences between groups.

A $p$-value $<0.05$ was considered statistically significant for all analyses.

All analyses were performed with the statistical software SAS 9.4 (SAS Institute, Cary, NC, USA).

\subsection{Surgical Technique}

Liver resection was performed either open or laparoscopically according to patients and tumor characteristics; regardless of the chosen approach, all patients underwent intraoperative ultrasound for tumor and vascular mapping.

CUSA ${ }^{\circledR}$ Excel/CUSA ${ }^{\circledR}$ Excel + Cavitron Ultrasonic Surgical Aspirator System (Integra, Dublin, Ireland) and Ultracision Harmonic scalpel (Ethicon Endo-Surgery, Cincinnati, OH, 
USA) were used for parenchymal transection, while hemostasis and biliostasis on the liver cut surface were achieved using metallic clips, Hemolock or non-absorbable sutures.

Pedicle clamping was not routinely applied; when needed, it was performed intermittently, with no longer than $15 \mathrm{~min}$ of clamping time and at least 5 min of release [23].

\subsection{Ablative Technique}

The majority of ablated patients underwent percutaneous ultrasound-guided radiofrequency ablation, and selected cases (i.e., perivascular lesions) received microwave coagulation therapy: the most commonly used electrode was a LeVeen Needle Electrode with an insulated 17-gauge outer needle and retractable curved electrodes (model 70 and model 90 Starburst XL needles, RITA Medical Systems, Mountain View, CA, USA; LeVeen needle electrode, Boston Scientific, Boston, MA, USA), while some patients were treated by an internally cooled electrode (Cool-Tip RF Electrode; Radionics, Burlington, MA, USA).

\section{Results}

Our starting population was composed of 688 cirrhotic patients: of these, 389 underwent SR and 299 received PA as first-line treatment between 2010 and 2016. One-hundredeighty-eight patients (93 resections and 95 ablations) were excluded as they presented multinodular disease. Other 125 patients (42 resections and 83 ablations) were excluded because of previous HCC diagnosis and/or treatment on past medical history. Eighty-eight patients (42 resections and 46 ablations) were excluded for a $<1$-year follow-up, so our population of 287 patients was composed of 75 ablated and 212 resected HCC. Preoperative imaging was not available for 118 resected and 21 ablated patients; 8 resected patients who presented macrovascular infiltration (Li-RADS-TIV) were furtherly excluded after imaging review. Our final population was composed of 140 patients: 86 resected and 54 ablated HCC. The workflow of patient selection is depicted in Figure 1.

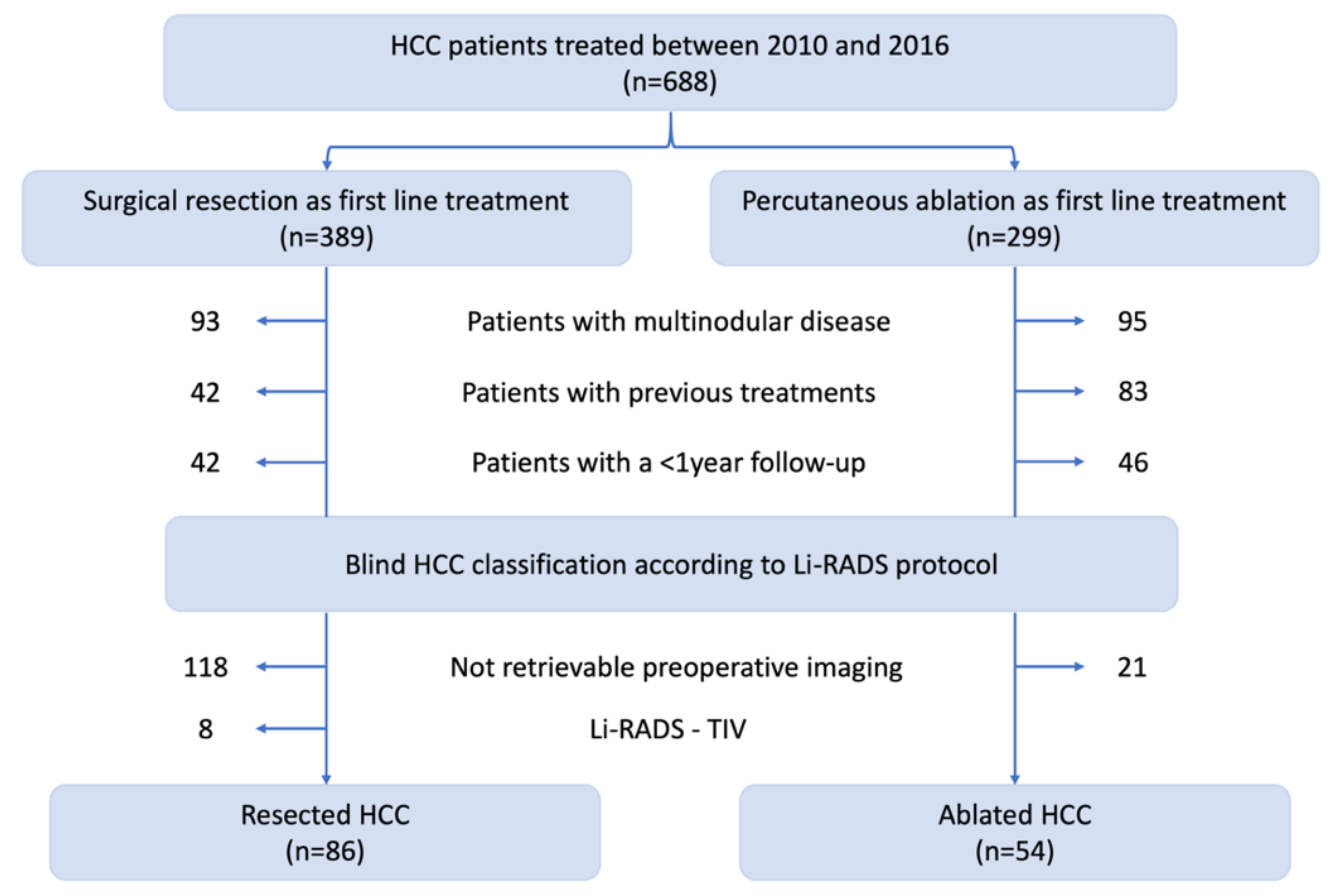

Figure 1. Flowchart for patient selection. 


\subsection{Patients and HCC Characteristics}

Clinical and demographical data of the study population are summarized in Table 1.

Table 1. Patients' characteristics before $(\mathrm{N}=140)$ and after $(\mathrm{N}=50)$ propensity score, by type of treatment.

\begin{tabular}{|c|c|c|c|c|c|c|c|}
\hline \multirow{2}{*}{ Variable } & \multirow{2}{*}{ Level } & \multicolumn{3}{|c|}{ Before Propensity Score } & \multicolumn{3}{|c|}{ After Propensity Score } \\
\hline & & SR $(N=86)$ & $P A(N=54)$ & $p^{1}$ & SR $(N=25)$ & PA (N = 25) & $p^{2}$ \\
\hline Age (year), median (IQR) & & $67(62,74)$ & $71(59,76)$ & 0.37 & $66(62,73)$ & $71(59,76)$ & 0.64 \\
\hline MELD score, median (IQR) & & $8(7,9)$ & $9(8,10)$ & 0.071 & $8(7,9)$ & $10(7,11)$ & 0.062 \\
\hline PLT, median (IQR) & & $159(109,229)$ & $98(78,139)$ & $<0.001$ & $126(100,159)$ & $106(79,171)$ & * \\
\hline ALT, median (IQR) & & $36(26,73)$ & $43(26,86)$ & 0.51 & $30(23,56)$ & $49(24,91)$ & 0.31 \\
\hline INR, median (IQR) & & $1.10(1.04,1.20)$ & $1.17(1.10,1.33)$ & 0.002 & $1.10(1.06,1.16)$ & $1.19(1.06,1.38)$ & 0.074 \\
\hline Bilirubin, median (IQR) & & $0.71(0.50,1.03)$ & $0.92(0.70,1.28)$ & 0.013 & $1.03(0.64,1.19)$ & $0.82(0.70,1.13)$ & 0.98 \\
\hline Albumin, median (IQR) & & $3.85(3.42,4.08)$ & $3.80(3.43,4.07)$ & 0.82 & $4.00(3.49,4.32)$ & $3.80(3.52,4.10)$ & 0.91 \\
\hline $\begin{array}{l}\text { Nodule Size (mm), median } \\
\text { (IQR) }\end{array}$ & & $30(24,45)$ & $18(15,22)$ & $<0.001$ & $23(20,27)$ & $20(17,24)$ & * \\
\hline Sex, N (\%) & $\begin{array}{c}\text { Men } \\
\text { Women }\end{array}$ & $\begin{array}{l}71(82.6) \\
15(17.4)\end{array}$ & $\begin{array}{l}37(68.5) \\
17(31.5)\end{array}$ & 0.086 & $\begin{array}{l}17(68.0) \\
8(32.0)\end{array}$ & $\begin{array}{l}21(84.0) \\
4(16.0)\end{array}$ & 0.32 \\
\hline Child score, N (\%) & $\begin{array}{l}\text { A } \\
\text { B }\end{array}$ & $\begin{array}{l}66(86.8) \\
10(13.2)\end{array}$ & $\begin{array}{l}38(79.2) \\
10(20.8)\end{array}$ & 0.38 & $\begin{array}{l}21(87.5) \\
3(12.5)\end{array}$ & $\begin{array}{l}18(75.0) \\
6(25.0)\end{array}$ & 0.46 \\
\hline ALBI score, N (\%) & $\begin{array}{l}\text { Grade I } \\
\text { Grade II } \\
\text { Grade III }\end{array}$ & $\begin{array}{c}35(40.7) \\
49(57.0) \\
2(2.3)\end{array}$ & $\begin{array}{c}16(32.7) \\
30(61.2) \\
3(6.1)\end{array}$ & 0.40 & $\begin{array}{c}11(44.0) \\
14(56.0) \\
0(0.0)\end{array}$ & $\begin{array}{c}10(40.0) \\
14(56.0) \\
1(4.0)\end{array}$ & 1.00 \\
\hline a-FP, N (\%) & $\begin{array}{c}\leq 5 \\
>5-22 \\
>22\end{array}$ & $\begin{array}{l}24(34.3) \\
27(38.6) \\
19(27.1)\end{array}$ & $\begin{array}{l}19(39.6) \\
16(33.3) \\
13(27.1)\end{array}$ & 0.81 & $\begin{array}{l}9(36.0) \\
9(36.0) \\
7(28.0)\end{array}$ & $\begin{array}{l}10(40.0) \\
8(32.0) \\
7(28.0)\end{array}$ & * \\
\hline \multirow[t]{2}{*}{$\begin{array}{c}\text { Presence of satellitosis, } \\
\text { N }(\%)\end{array}$} & No & $71(82.6)$ & $51(94.4)$ & 0.074 & $23(92.0)$ & $23(92.0)$ & * \\
\hline & Yes & $15(17.4)$ & $3(5.6)$ & & $2(8.0)$ & $2(8.0)$ & \\
\hline Type of nodule, N (\%) & $\begin{array}{l}\text { Superficial } \\
\text { Deep }\end{array}$ & $\begin{array}{l}45(52.3) \\
41(47.7)\end{array}$ & $\begin{array}{l}30(55.6) \\
24(44.4)\end{array}$ & 0.71 & $\begin{array}{l}18(72.0) \\
7(28.0)\end{array}$ & $\begin{array}{l}17(68.0) \\
8(32.0)\end{array}$ & 0.76 \\
\hline Li-RADS, N (\%) & $\begin{array}{l}\text { Li-RADS-3 } \\
\text { Li-RADS-4 } \\
\text { Li-RADS-5 }\end{array}$ & $\begin{array}{c}8(9.3) \\
15(17.4) \\
63(73.3)\end{array}$ & $\begin{array}{l}7(13.0) \\
12(22.2) \\
35(64.8)\end{array}$ & 0.56 & $\begin{array}{c}5(20.0) \\
4(16.0) \\
16(64.0)\end{array}$ & $\begin{array}{c}3(12.0) \\
3(12.0) \\
19(76.0)\end{array}$ & 0.68 \\
\hline \multicolumn{8}{|l|}{ Outcomes } \\
\hline $\begin{array}{l}\text { Hospital-stay (days), } \\
\text { median (IQR) }\end{array}$ & & $8(6,12)$ & $4(3,5)$ & $<0.001$ & $7(6,10)$ & $3(3,5)$ & $<0.001$ \\
\hline Complications, N (\%) & $\begin{array}{c}\text { None } \\
\text { Clavien 1-2 } \\
\text { Clavien 3-5 }\end{array}$ & $\begin{array}{l}51(59.3) \\
24(27.9) \\
11(12.8)\end{array}$ & $\begin{array}{c}47(87.0) \\
6(11.1) \\
1(1.9)\end{array}$ & 0.002 & $\begin{array}{c}19(76.0) \\
5(20.0) \\
1(4.0)\end{array}$ & $\begin{array}{c}20(80.0) \\
5(20.0) \\
0(0.0)\end{array}$ & 1.00 \\
\hline Comprehensive & Mean (SD) & $12.1(22.5)$ & $2.2(6.4)$ & $<0.001$ & $3.8(7.7)$ & $3.4(7.6)$ & 0.81 \\
\hline Complication Index & Median (IQR) & $0(0,20.9)$ & $0(0,0)$ & & $0(0,0)$ & $0(0,0)$ & \\
\hline R0 & $\begin{array}{l}\text { No } \\
\text { Yes }\end{array}$ & $\begin{array}{c}4(4.7) \\
82(95.3)\end{array}$ & - & - & $\begin{array}{c}0(0.0) \\
25(100.0)\end{array}$ & - & - \\
\hline
\end{tabular}

SR: surgical resection; PA: percutaneous ablation; IQR: interquartile range; PS: performance status. ${ }^{1}$ Wilcoxon p-value for continuous variables; Chi-square $p$-value for categorical variables; ${ }^{2}$ Wilcoxon $p$-value for continuous variables; Fisher's exact $p$-value for categorical variables; * Propensity score variables.

Resected patients presented lower bilirubin $(0.71 \mathrm{mg} / \mathrm{dL}$ vs. $0.92 \mathrm{mg} / \mathrm{dL} ; p=0.013)$, lower INR (1.10 vs. $1.17 ; p=0.001)$ and higher platelet count $(159.000 / \mu \mathrm{L}$ vs. $98.000 / \mu \mathrm{L}$; $p<0.001$ ) compared to patients who underwent PA, while albumin-bilirubin (ALBI) and Child Pugh scores were not significantly different between the two treatment groups.

Patients who underwent SR had larger nodules ( $30 \mathrm{~mm}$ vs. $18 \mathrm{~mm} ; p<0.001$ ) with higher prevalence of satellitosis, although not statistically significant (17.4\% vs. $5.7 \%$; $p=0.078$ ); both a-FP levels and distribution of Li-RADS classes did not significantly differ between and resected and ablated patients. Table S1 depicts the type of liver resections that were performed [23].

\subsection{Short-term Outcomes of PA and SR}

Patients who underwent $S R$ presented a higher incidence of clinically-relevant (ClavienDindo $\geq 3$ ) complications ( $12.8 \%$ vs. $1.9 \% ; p=0.002)$ and higher CCI (12.1 vs. $2.2 ; p<0.001)$ compared to patients treated by PA. 
Six patients who underwent PA developed a grade 1-2 postprocedural complication according to Clavien-Dindo classification: three patients had fever and were treated with antibiotics; two patients developed gastroparesis and vomiting requiring prokinetics medications and one patient experienced prolonged pain related to diaphragmatic irritation and was treated with opioid analgesics.

One patient who underwent PA developed an hemoperitoneum requiring endovascular embolization, resulting in a Clavien-Dindo 3a complication.

Twenty-four patients in the SR group developed a Clavien-Dindo grade 1-2 complication: postoperative ascites occurred in eight patients, that required diuretics; five patients developed fever treated with antibiotics; four patients had a pleural effusion requiring diuretics and respiratory physiotherapy; three patients complained of nausea and vomiting, requiring prokinetics medications; two patients developed a mild pneumonia and two other patients had a superficial surgical site infection.

Nine patients who underwent SR developed a Clavien-Dindo grade 3-4 complication: two patients developed a deep surgical site infection treated by percutaneous drainage, resulting in a Clavien-Dindo 3a complication; an ISGLS grade B postoperative biliary fistula requiring endoscopic management occurred in two patients, resulting in a Clavien-Dindo 3a complication; three patients underwent relaparotomy for hemoperitoneum yielding a Clavien-Dindo 4a complication and two patients developed a grade $C$ post-hepatectomy liver failure according ISGLS definition, resulting in a Clavien-Dindo $4 \mathrm{~b}$ complication. Two fatalities occurred after SR, yielding in a $2.3 \%$ postoperative mortality.

Finally, SR resulted in a significantly longer hospital stay compared to PA ( 8 days vs. 4 days; $p<0.001$ ).

\subsection{Survival Analysis}

Median follow-up lasted 5 years (IQR: 2.1-7.1 years) in the resected and 4.5 years (IQR: 3.1-5.4 years) in the ablated population.

Considering the general population, SR offered significantly better OS $(87.1 \%, 73.6 \%$ and $68.3 \%$ vs. $94.4 \%, 81.2 \%$ and $52.2 \% 1-, 3$ - and 5 -year OS; $p=0.049)$, RFS $(82.2 \%, 60.7 \%$ and $42.5 \%$ vs. $52.5 \%, 34.6 \%$ and $29.8 \% 1-, 3$ - and 5-year RFS; $p=0.002$ ) and lower incidence of local recurrence $(2.4 \%, 7.1 \%$ and $8.2 \%$ vs. $37.0 \%, 42.6 \%$ and $44.4 \% 1-, 3$ - and 5 -year CIF of local recurrence; $p<0.001$ ) compared to PA (Figure 2).

The management of local and intrahepatic recurrences that were diagnosed during the follow-up period are resumed in Figure S1 (after PA as first-line treatment) and Figure S2 (after SR as firs-line treatment).

\subsection{Survival Analysis of Li-RADS Subclasses}

The second step of the analysis focused on oncological outcomes of SR and PA between two subsets of patients: those with a Li-RADS-3/4 and those with a Li-RADS-5 HCC (Figure 3).

Median follow-up lasted 5.9 years (IQR: 1.6-9.5 years) in the resected and 4.5 years (IQR: 3.5-5.4 years) in the ablated population.

OS did not significantly differ between resected vs. ablated patients in Li-RADS-3/4 subclasses $(87.0 \%, 81.8 \%$ and $71.6 \%$ vs. $100.0 \%, 88.9 \%$ and $63.0 \% 1-, 3-$ and 5 -year OS; $p=0.625)$ while it was significantly better in Li-RADS-5 HCC after SR $(87.1 \%, 70.9 \%$ and $67.1 \%$ vs. $91.4 \%, 77.1 \%$ and $46.2 \% 1$-, 3 - and 5 -year OS; $p=0.035$ ).

The abovementioned statistically significant difference in RFS between resected and ablated patients was no longer evident in Li-RADS-3/4 (81.0\%, $56.7 \%$ and $34.8 \%$ vs. $62.7 \%$, $34.8 \%$ and $34.8 \% 1-, 3$ - and 5-year RFS; $p=0.758)$ HCCs, while it was retained in Li-RADS5 subclass $(82.7 \%, 62.3 \%$ and $45.0 \%$ vs. $47.1 \%, 34.2 \%$ and $27.0 \% 1-, 3-$ and 5 -year RFS; $p<0.001)$.

The incidence of local recurrence was significantly lower after SR for both Li-RADS$3 / 4(0.0 \%, 0.0 \%$ and $4.3 \%$ vs. $31.6 \%, 36.8 .6 \%$ and $36.8 \% 1-, 3-$, and 5 -year CIF of local 
recurrence; $p=0.007)$ and Li-RADS-5 nodules $(3.2 \%, 9.7 \%$ and $9.7 \%$ vs. $40.0 \%, 45.7 \%$ and $48.6 \%$ 1-, 3- and 5-year CIF of local recurrence; $p<0.001$ ).

(A)

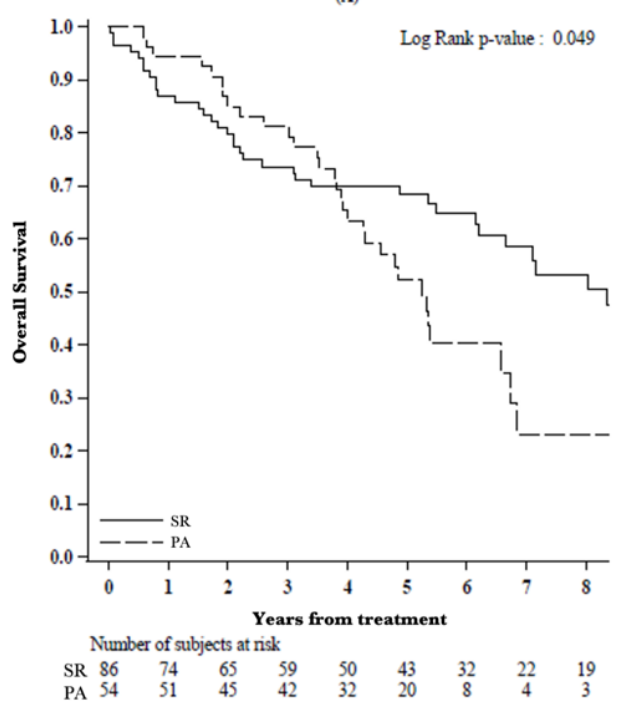

(C)

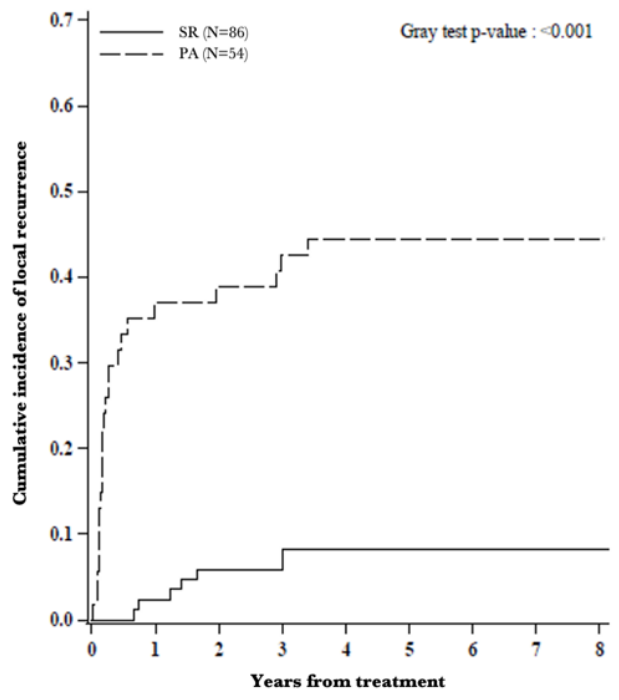

(B)

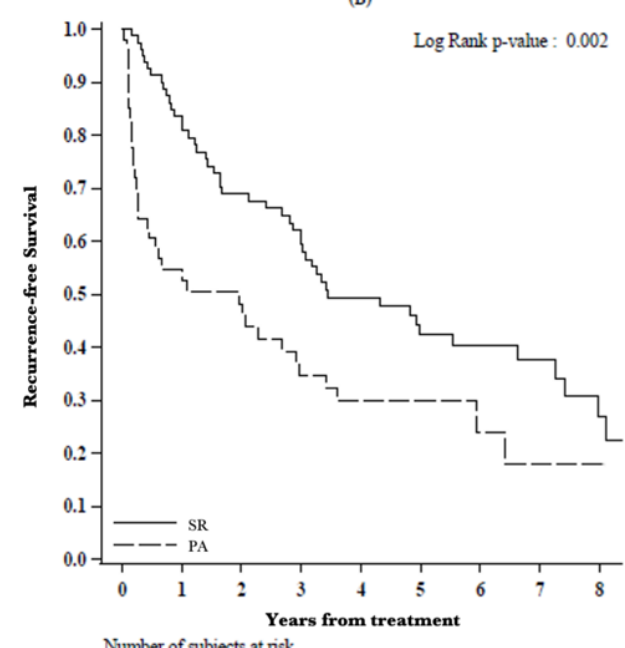

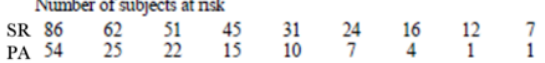

Figure 2. (A) Overall survival, (B) recurrence-free survival and (C) cumulative incidence of local recurrence by type of treatment $(\mathrm{N}=140)$.

\subsection{Oncological Outcomes of PS-M Population}

Median follow-up lasted 7.1 years (IQR: 3.4-9.6 years) in the resected and 5.3 years (IQR: 3.4-9.6 years) in the ablated population.

After PS matching, both OS (96.0\%, 87.5\% and 78.8\% vs. $96.0 \%, 84.0 \%$ and $53.7 \%$ 1-, 3 - and 5 -year OS; $p=0.025)$, RFS $(87.7 \%, 50.1 \%$ and $41.3 \%$ vs. $46.2 \%, 32.3 \%$ and $32.3 \%$ $1-, 3$ - and 5-year RFS; $p=0.049)$ and the incidence of local recurrence $(0.0 \%, 12.0 \%$ and $12.0 \%$ vs. $48.0 \%, 56.0 \%$ and $56.0 \% 1-, 3$ - and 5 -year CIF of local recurrence; $p<0.001$ ) were significantly better after SR compared to PA (Figure S3).

Focusing on oncological results in different Li-RADS classes, we highlighted better OS $(93.8 \%, 80.8 \%$ and $80.8 \%$ vs. $94.7 \%, 84.2 \%$ and $43.7 \% 1-, 3-$ and 5 -year OS; $p=0.009)$, RFS $(93.8 \%, 46.9 \%$ and $40.2 \%$ vs. $40.2 \%, 28.7 \%$ and $28.7 \% 1-, 3-$ and $5-$ year RFS; $p=0.038)$ and lower incidence of local recurrence $(0.0 \%, 18.8 \%$ and $18.8 \%$ vs. $52.6 \%, 63.2 \%$ and $63.2 \% 1-$, 3 - and 5-year CIF of local recurrence; $p=0.005$ ) after SR in Li-RADS-5 nodules. 
On the other hand, OS, RFS and CIF of local recurrence in Li-RADS-3/4 HCCs did not significantly differ between the two treatment groups (Figure S4).
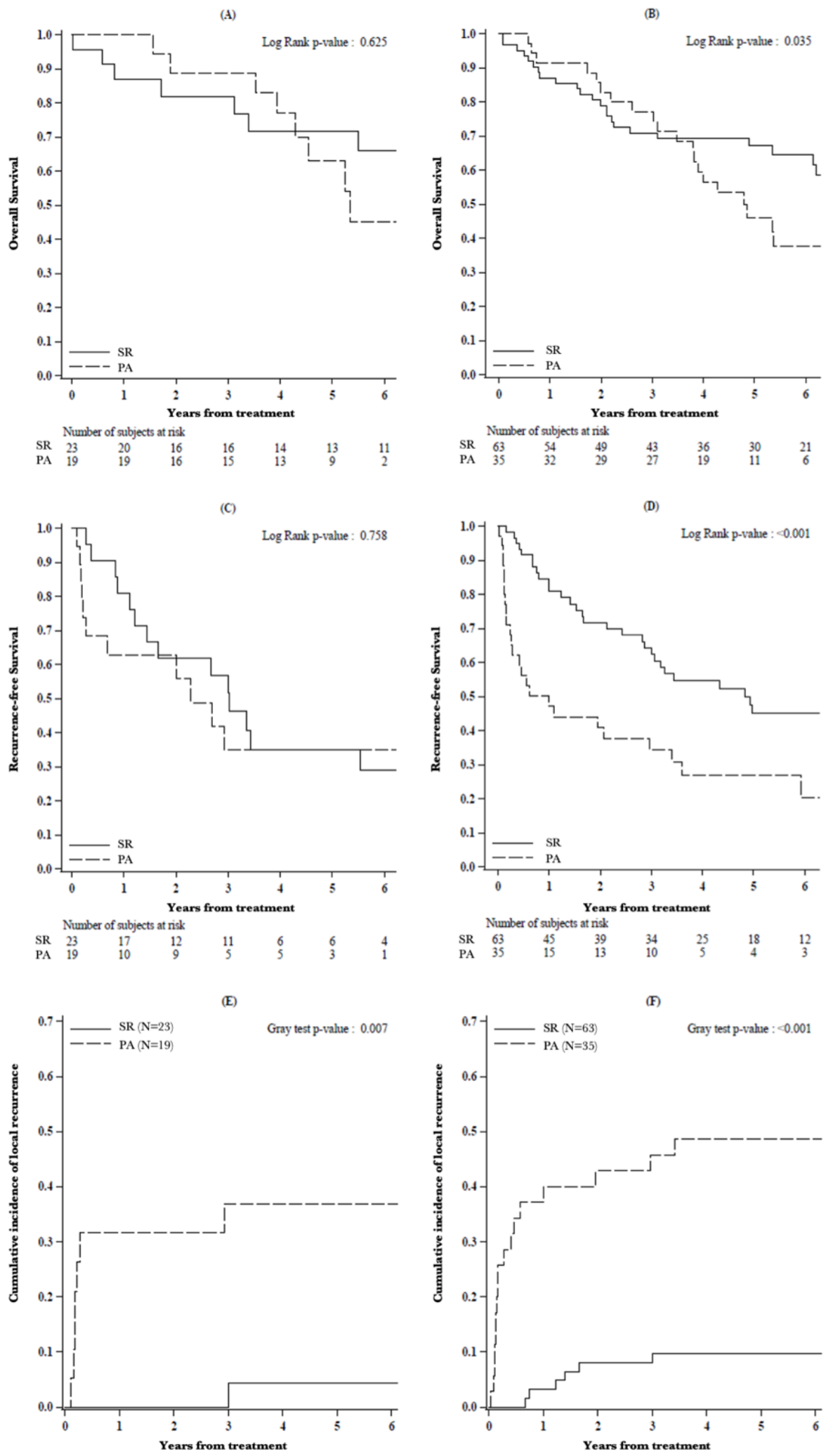

Figure 3. (A,B) Overall survival, (C,D) recurrence-free survival and (E,F) cumulative incidence of local recurrence in patients with nodule classified as (A,C,E) Li-RADS-3/4 and (B,D,F) Li-RADS-5, by type of treatment. 


\subsection{Pathological Analysis of Resected Specimens}

We achieved a R0 resection in 95\% of HCCs. Focusing on the association between pathological features and Li-RADS subclasses, we documented a slightly higher frequency of G3 tumors ( $31.7 \%$ vs. $17.4 \% ; p=0.14)$, microvascular invasion $(47.6 \%$ vs. $26.1 \%$; $p=0.07)$ and satellitosis $(20.6 \%$ vs. $8.7 \%$; $p=0.33)$ in Li-RADS-5 nodules.

\section{Discussion}

The best approach to single HCC is still debated: several studies reported similar oncological outcomes after PA and SR [8-10], although many pieces of evidence support the superiority of surgery [11-13].

PA offers a minimally invasive approach that may grant acceptable oncological results with an average 5-year OS of 30-76\% and a 5-year RFS of 14-49\% [10,12,13,24-27]; compared to SR, PA shows better complication rates [13,28], but its oncological efficacy might be impaired by tumor location $[11,29,30]$ and size $[31,32]$.

On the other hand, SR offers a 5-year OS of $62-86 \%$ and a 5 -year RFS of $41-82 \%$ [13,33-35], but bears higher complication rates compared to PA; moreover, SR could be precluded by impaired liver function that may limit extended parenchymal resections [34,36].

In this study we focused on a highly selected subset of patients presenting with a single treatment-naïve HCC without macrovascular invasion, approached by SR or PA.

As already highlighted in other series comparing surgery and ablation for HCC [11-13,28,34-36], SR showed an increased incidence of clinically-relevant complications, depicted by a higher CCI. Despite that, it should be noticed that the mean CCI of 12.2 in this surgical population of cirrhotic patients did not differ remarkably from the recently proposed ideal benchmarks of postoperative outcomes after liver resections [37].

Focusing on oncological outcomes, the analysis of our general population highlighted better OS, RFS and lower incidence of local recurrence after SR, confirming the results from other series [11-13].

Several studies pointed out that tumor size and location might affect the efficacy of ablative techniques: in fact, it has been proven that PA performs better in HCC nodules $\leq 3 \mathrm{~cm}$, as documented by the $70 \%$ complete necrosis after histological analysis of transplant specimens [38]. Likewise, PA for subcapsular [39,40] or perivascular [41,42] tumors seem to achieve worse oncological results. On this behalf, it but must be noticed how mean tumor size of the ablated nodules was $18 \mathrm{~mm}$ and $20 \mathrm{~mm}$ before and after PS-M, respectively, and the distribution of tumor location did not significantly differ among the two treatment groups.

The impact of Li-RADS classification on the oncological outcomes of the two treatments in our unmatched population highlighted better OS and RFS in Li-RADS- 5 nodules that underwent SR, while these differences were not evident for Li-RADS-3/4 classes. Likewise, our analysis showed higher incidence of local recurrence after PA regardless of Li-RADS classification.

We further performed a PS-M analysis, looking for stronger validation of the abovementioned results: such model was build considering the role of tumor size [43], satellitosis [44], a-FP levels [45] and liver function [46] on the oncological aggressiveness of HCC, in order to balance any confounding differences between the two treatment groups. Despite preliminary analysis of the baseline characteristics in pre-propensity population documented statistically significant higher INR and bilirubin levels in the ablated patients, these differences were not clinically relevant as their values were between the normal range in both treatment groups. On the other hand, the lower platelet count in PA group was considered as a significant indicator for portal hypertension, and incorporated in PS-M, as well as tumor related features such as size, a-FP and satellitosis.

After PS-M, we confirmed better OS, RFS and lower incidence of local recurrence after $\mathrm{SR}$, documenting a deeper influence of Li-RADS classification on the oncological outcomes of the two treatments: in fact, SR resulted in better OS and RFS with lower incidence 
of local recurrence only for Li-RADS-5 nodules, while these outcomes did not differ for Li-RADS-3/4 subclasses.

These results might be explained by a more aggressive behavior of Li-RADS-5 HCCs compared to Li-RADS-3/4 nodules: such different outcomes could possibly rely on the association of Li-RADS- 5 class with worse histological features. Despite the diagnostic accuracy of Li-RADS classification has been widely validated [7,47], up to now there are few reports focusing on its correlation with tumor differentiation and histology [48]. Even though the analysis on our resection specimens did not highlight any statically significant correlation between Li-RADS- 5 class and unfavorable histological features, we depicted a higher frequency of these high-risk characteristics in Li-RADS- 5 nodules, especially for microvascular invasion. Such radiological/histological correlation has never been investigated so far, and deserves further efforts on larger populations.

The main limitation of this study is represented by its retrospective nature, which may imply selection and indication biases, although Li-RADS classification did not influence neither patient selection nor management as it was retrospectively and blindly applied after treatment.

Another limitation is the relatively small study population, partially related to the loss of several cases for not-retrievable preoperative imaging (as many patients were referred by general practitioners or other centers after diagnostic workup); despite that, we should keep in mind that this analysis focused on a highly selected subclass of patients from a single center, which represented per se less than a half of the starting population.

\section{Conclusions}

This is the first analysis focusing on the impact of Li-RADS classification on the oncological results of SR vs. PA for single HCC that has been reported so far.

Despite a relatively higher complication rate after surgery, our findings support the superiority of SR over PA confirming the conclusions from other groups, and suggest a new potential role of Li-RADS classification in clinical decision making, shifting from a diagnostic to a prognostic tool.

Following these preliminary observations, SR should be especially supported in those patients bearing a Li-RADS- 5 HCC in order to achieve better oncological results, whenever feasible. On the other hand, PA seems to grant similar outcomes compared to SR in LiRADS-3/4 nodules, and should be considered as a valuable option for those cases requiring challenging surgeries or in less compensated patients.

Although promising, these findings should be cautiously applied to clinical practice, and several parameters (a-FP levels, liver functional reserve, tumor location) beside from Li-RADS subclass must always be evaluated in order to tailor the therapeutic approach to the individual patient and clinical context.

The results of this exploratory analysis should be verified on larger multicentric populations and hopefully validated by randomized cohort studies.

Supplementary Materials: The following are available online at https:/ /www.mdpi.com/article/10 .3390 / cancers13071671/s1, Table S1: Surgical technique before propensity score matching $(\mathrm{N}=86)$; Figure S1: Management of local and intrahepatic recurrences after percutaneous ablation; Figure S2: Management of local and intrahepatic recurrences after surgical resection; Figure S3: (A) Overall survival, (B) recurrence-free survival and (C) cumulative incidence of local recurrence by type of treatment, among patients after propensity score $(\mathrm{N}=50)$; Figure $S 4$ : $(A, B)$ Overall survival, $(C, D)$ recurrence- free survival and $(\mathrm{E}, \mathrm{F})$ cumulative incidence of local recurrence in patients with nodule classified as (A,C,E) Li-RADS-3/4 and (B,D,F) Li-RADS-5, by type of treatment, among patients after propensity score.

Author Contributions: Conceptualization, L.C. and S.D.S.; methodology, L.C., S.D.S. and V.B.; formal analysis, S.F. and V.B.; data curation, L.C., R.D.C., B.T. and A.V.; writing-original draft preparation, L.C.; writing-review and editing, L.C., S.D.S., R.D.C. and A.L.; supervision, A.R., A.V. and L.D.C. All authors have read and agreed to the published version of the manuscript. 
Funding: This research received no external funding.

Institutional Review Board Statement: The study was conducted according to the guidelines of the Declaration of Helsinki; local ethical committees' review of the protocol deemed that formal approval was not required owing to the retrospective, observational and anonymous nature of this study.

Informed Consent Statement: Informed consent was obtained from all subjects involved in the study.

Data Availability Statement: Data will be available upon request to the corresponding author.

Conflicts of Interest: The authors declare no conflict of interest.

\section{References}

1. Bray, F.; Ferlay, J.; Soerjomataram, I.; Siegel, R.L.; Torre, L.A.; Jemal, A. Global cancer statistics 2018: GLOBOCAN estimates of incidence and mortality worldwide for 36 cancers in 185 countries. CA Cancer J. Clin. 2018, 68, 394-424. [CrossRef]

2. Lafaro, K.J.; Demirjian, A.N.; Pawlik, T.M. Epidemiology of Hepatocellular Carcinoma. Surg. Oncol. Clin. N. Am. 2015, 24, 1-17. [CrossRef] [PubMed]

3. Gheorghe, G.; Stoian, A.P.; Gaman, M.-A.; Socea, B.; Neagu, T.P.; Stanescu, A.M.A.; Bratu, O.G.; Mischianu, D.L.D.; Suceveanu, A.I.; Diaconu, C.C. The Benefits and Risks of Antioxidant Treatment in Liver Diseases. Rev. Chim. 2019, 70, 651-655. [CrossRef]

4. European Association for the Study of the Liver. Electronic address eee, European Association for the Study of the L. EASL Clinical Practice Guidelines: Management of hepatocellular carcinoma. J. Hepatol. 2018, 69, 182-236. [CrossRef]

5. Marrero, J.A.; Kulik, L.M.; Sirlin, C.B.; Zhu, A.X.; Finn, R.S.; Abecassis, M.M.; Roberts, L.R.; Heimbach, J.K. Diagnosis, Staging, and Management of Hepatocellular Carcinoma: 2018 Practice Guidance by the American Association for the Study of Liver Diseases. Clin. Liver Dis. 2019, 13, 1. [CrossRef]

6. $\quad$ Chernyak, V.; Fowler, K.J.; Kamaya, A.; Kielar, A.Z.; Elsayes, K.M.; Bashir, M.R.; Kono, Y.; Do, R.K.; Mitchell, D.G.; Singal, A.G.; et al. Liver Imaging Reporting and Data System (LI-RADS) Version 2018: Imaging of Hepatocellular Carcinoma in At-Risk Patients. Radiology 2018, 289, 816-830. [CrossRef] [PubMed]

7. Tang, E.S.-T.; Hall, G.; Yu, D.; Menard, A.; Hopman, W.; Nanji, S. Predictors and Cumulative Frequency of Hepatocellular Carcinoma in High and Intermediate LI-RADS Lesions: A Cohort Study from a Canadian Academic Institution. Ann. Surg. Oncol. 2019, 26, 2560-2567. [CrossRef] [PubMed]

8. Forner, A.; Reig, M.; Bruix, J. Hepatocellular carcinoma. Lancet 2018, 391, 1301-1314. [CrossRef]

9. Pompili, M.; Saviano, A.; de Matthaeis, N.; Cucchetti, A.; Ardito, F.; Federico, B.; Brunello, F.; Pinna, A.D.; Giorgio, A.; Giulini, S.M.; et al. Long-term effectiveness of resection and radiofrequency ablation for single hepatocellular carcinoma $\leq 3 \mathrm{~cm}$. Results of a multicenter Italian survey. J. Hepatol. 2013, 59, 89-97. [CrossRef] [PubMed]

10. Wang, J.-H.; Wang, C.-C.; Hung, C.-H.; Chen, C.-L.; Lu, S.-N. Survival comparison between surgical resection and radiofrequency ablation for patients in BCLC very early/early stage hepatocellular carcinoma. J. Hepatol. 2012, 56, 412-418. [CrossRef] [PubMed]

11. Lee, S.; Kang, T.W.; Cha, D.I.; Song, K.D.; Lee, M.W.; Rhim, H.; Lim, H.K.; Sinn, D.H.; Kim, J.M.; Kim, K. Radiofrequency ablation vs. surgery for perivascular hepatocellular carcinoma: Propensity score analyses of long-term outcomes. J. Hepatol. 2018, 69, 70-78. [CrossRef] [PubMed]

12. Liu, P.-H.; Hsu, C.-Y.; Hsia, C.-Y.; Lee, Y.-H.; Huang, Y.-H.; Chiou, Y.-Y.; Lin, H.-C.; Huo, T.-I. Surgical Resection Versus Radiofrequency Ablation for Single Hepatocellular Carcinoma $\leq 2 \mathrm{~cm}$ in a Propensity Score Model. Ann. Surg. 2016, 263, 538-545. [CrossRef]

13. Feng, K.; Yan, J.; Li, X.; Xia, F.; Ma, K.; Wang, S.; Bie, P.; Dong, J. A randomized controlled trial of radiofrequency ablation and surgical resection in the treatment of small hepatocellular carcinoma. J. Hepatol. 2012, 57, 794-802. [CrossRef] [PubMed]

14. Di Sandro, S.; Sposito, C.; Lauterio, A.; Najjar, M.; Busset, M.D.D.; Buscemi, V.; Reyes, M.F.; De Carlis, R.; Mazzaferro, V.; De Carlis, L. Proposal of Prognostic Survival Models before and after Liver Resection for Hepatocellular Carcinoma in Potentially Transplantable Patients. J. Am. Coll. Surg. 2018, 226, 1147-1159. [CrossRef] [PubMed]

15. Lai, Q.; Vitale, A.; Iesari, S.; Finkenstedt, A.; Mennini, G.; Spoletini, G.; Hoppe-Lotichius, M.; Vennarecci, G.; Manzia, T.M.; Nicolini, D.; et al. Intention-to-treat survival benefit of liver transplantation in patients with hepatocellular cancer. Hepatology 2017, 66, 1910-1919. [CrossRef]

16. Di Sandro, S.; Bagnardi, V.; Cucchetti, A.; Lauterio, A.; De Carlis, R.; Benuzzi, L.; Danieli, M.; Botta, F.; Centonze, L.; Najjar, M.; et al. From a Philosophical Framework to a Valid Prognostic Staging System of the New "Comprehensive Assessment" for Transplantable Hepatocellular Carcinoma. Cancers 2019, 11, 741. [CrossRef] [PubMed]

17. Von Elm, E.; Altman, D.G.; Egger, M.; Pocock, S.J.; Gøtzsche, P.C.; Vandenbroucke, J.P.; Initiative, F.T.S. The Strengthening the Reporting of Observational Studies in Epidemiology (STROBE) Statement: Guidelines for Reporting Observational Studies. PLoS Med. 2007, 4, 296. [CrossRef] [PubMed]

18. Dindo, D.; Demartines, N.; Clavien, P.-A. Classification of Surgical Complications: A new proposal with evaluation in a cohort of 6336 patients and results of a survey. Ann. Surg. 2004, 240, 205-213. [CrossRef] [PubMed]

19. Slankamenac, K.; Graf, R.; Barkun, J.; Puhan, M.A.; Clavien, P.-A. The Comprehensive Complication Index. Ann. Surg. 2013, 258, 1-7. [CrossRef] [PubMed] 
20. Koch, M.; Garden, O.J.; Padbury, R.; Rahbari, N.N.; Adam, R.; Capussotti, L.; Fan, S.T.; Yokoyama, Y.; Crawford, M.; Makuuchi, M.; et al. Bile leakage after hepatobiliary and pancreatic surgery: A definition and grading of severity by the International Study Group of Liver Surgery. Surgery 2011, 149, 680-688. [CrossRef]

21. Rahbari, N.N.; Garden, O.J.; Padbury, R.; Brooke-Smith, M.; Crawford, M.; Adam, R.; Koch, M.; Makuuchi, M.; Dematteo, R.P.; Christophi, C.; et al. Posthepatectomy liver failure: A definition and grading by the International Study Group of Liver Surgery (ISGLS). Surgery 2011, 149, 713-724. [CrossRef] [PubMed]

22. Chan, A.C.Y.; Chan, S.C.; Chok, K.S.H.; Cheung, T.T.; Chiu, D.W.; Poon, R.T.P.; Fan, S.T.; Lo, C.M. Treatment strategy for recurrent hepatocellular carcinoma: Salvage transplantation, repeated resection, or radiofrequency ablation? Liver Transplant. 2013, 19, 411-419. [CrossRef] [PubMed]

23. Di Sandro, S.; Bagnardi, V.; Najjar, M.; Buscemi, V.; Lauterio, A.; De Carlis, R.; Danieli, M.; Pinotti, E.; Benuzzi, L.; De Carlis, L. Minor laparoscopic liver resection for Hepatocellular Carcinoma is safer than minor open resection, especially for less compensated cirrhotic patients: Propensity score analysis. Surg. Oncol. 2018, 27, 722-729. [CrossRef] [PubMed]

24. Kim, Y.-S.; Lim, H.K.; Rhim, H.; Lee, M.W.; Choi, D.; Lee, W.J.; Paik, S.W.; Koh, K.C.; Lee, J.H.; Choi, M.S.; et al. Ten-year outcomes of percutaneous radiofrequency ablation as first-line therapy of early hepatocellular carcinoma: Analysis of prognostic factors. J. Hepatol. 2013, 58, 89-97. [CrossRef]

25. Kang, T.W.; Kim, J.M.; Rhim, H.; Lee, M.W.; Kim, Y.-S.; Lim, H.K.; Choi, D.; Song, K.D.; Kwon, C.H.D.; Joh, J.-W.; et al. Small Hepatocellular Carcinoma: Radiofrequency Ablation versus Nonanatomic Resection-Propensity Score Analyses of Long-term Outcomes. Radiology 2015, 275, 908-919. [CrossRef]

26. Lee, G.C.; Ferrone, C.R.; Vagefi, P.A.; Uppot, R.N.; Tanabe, K.K.; Lillemoe, K.D.; Blaszkowsky, L.S.; Qadan, M. Surgical resection versus ablation for early-stage hepatocellular carcinoma: A retrospective cohort analysis. Am. J. Surg. 2019, 218, 157-163. [CrossRef]

27. Uhlig, J.; Sellers, C.M.; Stein, S.M.; Kim, H.S. Radiofrequency ablation versus surgical resection of hepatocellular carcinoma: Contemporary treatment trends and outcomes from the United States National Cancer Database. Eur. Radiol. 2018, 29, $2679-2689$. [CrossRef]

28. Vitale, A.; Peck-Radosavljevic, M.; Giannini, E.G.; Vibert, E.; Sieghart, W.; Van Poucke, S.; Pawlik, T.M. Personalized treatment of patients with very early hepatocellular carcinoma. J. Hepatol. 2017, 66, 412-423. [CrossRef]

29. Mulier, S.; Ni, Y.; Jamart, J.; Ruers, T.; Marchal, G.; Michel, L. Local Recurrence After Hepatic Radiofrequency Coagulation. Ann. Surg. 2005, 242, 158-171. [CrossRef] [PubMed]

30. Ito, T.; Tanaka, S.; Iwai, S.; Takemura, S.; Hagihara, A.; Uchida-Kobayashi, S.; Shinkawa, H.; Nishioka, T.; Kawada, N.; Kubo, S. Outcomes of laparoscopic hepatic resection versus percutaneous radiofrequency ablation for hepatocellular carcinoma located at the liver surface: A case-control study with propensity score matching. Hepatol. Res. 2015, 46, 565-574. [CrossRef] [PubMed]

31. Tsuchiya, K.; Asahina, Y.; Tamaki, N.; Yasui, Y.; Hosokawa, T.; Ueda, K.; Nakanishi, H.; Itakura, J.; Kurosaki, M.; Enomoto, N.; et al. Risk factors for exceeding the Milan criteria after successful radiofrequency ablation in patients with early-stage hepatocellular carcinoma. Liver Transplant. 2013, 20, 291-297. [CrossRef] [PubMed]

32. Kutlu, O.C.; Chan, J.A.; Aloia, T.A.; Chun, Y.S.; Kaseb, A.O.; Passot, G.; Yamashita, S.; Vauthey, J.-N.; Conrad, C. Comparative effectiveness of first-line radiofrequency ablation versus surgical resection and transplantation for patients with early hepatocellular carcinoma. Cancer 2017, 123, 1817-1827. [CrossRef]

33. Huang, J.; Yan, L.; Cheng, Z.; Wu, H.; Du, L.; Wang, J.; Xu, Y.; Zeng, Y. A Randomized Trial Comparing Radiofrequency Ablation and Surgical Resection for HCC Conforming to the Milan Criteria. Ann. Surg. 2010, 252, 903-912. [CrossRef] [PubMed]

34. Liu, H.; Wang, Z.; Fu, S.; Li, A.; Pan, Z.; Zhou, W.; Lau, W.; Wu, M. Randomized clinical trial of chemoembolization plus radiofrequency ablation versus partial hepatectomy for hepatocellular carcinoma within the Milan criteria. Br. J. Surg. 2016, 103, 348-356. [CrossRef] [PubMed]

35. Kim, G.; Shim, J.H.; Kim, M.; Kim, S.Y.; Won, H.J.; Shin, Y.M.; Kim, P.N.; Kim, K.; Lee, S.; Lee, H.C. Radiofrequency ablation as an alternative to hepatic resection for single small hepatocellular carcinomas. Br. J. Surg. 2015, 103, 126-135. [CrossRef] [PubMed]

36. Fang, Y.; Chen, W.; Liang, X.; Li, D.; Lou, H.; Chen, R.; Wang, K.; Pan, H. Comparison of long-term effectiveness and complications of radiofrequency ablation with hepatectomy for small hepatocellular carcinoma. J. Gastroenterol. Hepatol. 2014, 29, 193-200. [CrossRef] [PubMed]

37. Rössler, F.; Sapisochin, G.; Song, G.; Lin, Y.-H.; Simpson, M.A.; Hasegawa, K.; Laurenzi, A.; Cabús, S.S.; Nunez, M.I.; Gatti, A.; et al. Defining Benchmarks for Major Liver Surgery. Ann. Surg. 2016, 264, 492-500. [CrossRef] [PubMed]

38. Pompili, M.; Mirante, V.G.; Rondinara, G.; Fassati, L.R.; Piscaglia, F.; Agnes, S.; Covino, M.; Ravaioli, M.; Fagiuoli, S.; Gasbarrini, G.; et al. Percutaneous ablation procedures in cirrhotic patients with hepatocellular carcinoma submitted to liver transplantation: Assessment of efficacy at explant analysis and of safety for tumor recurrence. Liver Transplant. 2005, 11, 1117-1126. [CrossRef]

39. Hori, T.; Nagata, K.; Hasuike, S.; Onaga, M.; Motoda, M.; Moriuchi, A.; Iwakiri, H.; Uto, H.; Kato, J.; Ido, A.; et al. Risk factors for the local recurrence of hepatocellular carcinoma after a single session of percutaneous radiofrequency ablation. J. Gastroenterol. 2003, 38, 977-981. [CrossRef] [PubMed]

40. Kang, T.W.; Lim, H.K.; Lee, M.W.; Kim, Y.-S.; Rhim, H.; Lee, W.J.; Paik, Y.H.; Kim, M.J.; Ahn, J.H. Long-term Therapeutic Outcomes of Radiofrequency Ablation for Subcapsular versus Nonsubcapsular Hepatocellular Carcinoma: A Propensity Score Matched Study. Radiology 2016, 280, 300-312. [CrossRef] 
41. Teratani, T.; Yoshida, H.; Shiina, S.; Obi, S.; Sato, S.; Tateishi, R.; Mine, N.; Kondo, Y.; Kawabe, T.; Omata, M. Radiofrequency ablation for hepatocellular carcinoma in so-called high-risk locations. Hepatology 2006, 43, 1101-1108. [CrossRef]

42. Komorizono, Y.; Oketani, M.; Sako, K.; Yamasaki, N.; Shibatou, T.; Maeda, M.; Kohara, K.; Shigenobu, S.; Ishibashi, K.; Arima, T. Risk factors for local recurrence of small hepatocellular carcinoma tumors after a single session, single application of percutaneous radiofrequency ablation. Cancer 2003, 97, 1253-1262. [CrossRef] [PubMed]

43. Wang, J.; Li, Q.; Sun, Y.; Zheng, H.; Cui, Y.; Li, H.; Zhou, H.; Hao, X. Clinicopathologic features between multicentric occurence and intrahepatic metastasis of multiple hepatocellular carcinomas related to HBV. Surg. Oncol. 2009, 18, 25-30. [CrossRef] [PubMed]

44. Poon, R.T.-P.; Fan, S.-T.; Wong, J. Risk Factors, Prevention, and Management of Postoperative Recurrence After Resection of Hepatocellular Carcinoma. Ann. Surg. 2000, 232, 10-24. [CrossRef] [PubMed]

45. Di Bisceglie, A.M.; Sterling, R.K.; Chung, R.T.; Everhart, J.E.; Dienstag, J.L.; Bonkovsky, H.L.; Wright, E.C.; Everson, G.T.; Lindsay, K.L.; Lok, A.S.; et al. Serum alpha-fetoprotein levels in patients with advanced hepatitis C: Results from the HALT-C Trial. J. Hepatol. 2005, 43, 434-441. [CrossRef] [PubMed]

46. Cucchetti, A.; Piscaglia, F.; Caturelli, E.; Benvegnù, L.; Vivarelli, M.; Ercolani, G.; Cescon, M.; Ravaioli, M.; Grazi, G.L.; Bolondi, L.; et al. Comparison of Recurrence of Hepatocellular Carcinoma After Resection in Patients with Cirrhosis to Its Occurrence in a Surveilled Cirrhotic Population. Ann. Surg. Oncol. 2008, 16, 413-422. [CrossRef]

47. LaRoia, S.T.; Yadav, K.; Rastogi, A.; Kumar, G.; Kumar, S.; Sarin, S.K. Diagnostic efficacy of dynamic liver imaging using qualitative diagnostic algorithm versus LI-RADS v2018 lexicon for atypical versus classical HCC lesions: A decade of experience from a tertiary liver institute. Eur. J. Radiol. Open 2020, 7, 100219. [CrossRef] [PubMed]

48. Granata, V.; Fusco, R.; Setola, S.V.; Picone, C.; Vallone, P.; Belli, A.; Incollingo, P.; Albino, V.; Tatangelo, F.; Izzo, F.; et al. Microvascular invasion and grading in hepatocellular carcinoma: Correlation with major and ancillary features according to LIRADS. Abdom. Radiol. 2019, 44, 2788-2800. [CrossRef] 